\title{
Review of: "Identifying and characterizing pesticide use on 9,000 fields of organic agriculture"
}

\author{
Amin Mojiri
}

Potential competing interests: The author(s) declared that no potential competing interests exist.

I have read the paper entitled "Identifying and characterizing pesticide use on 9,000 fields of organic agriculture" published by Nature Communication. It is a very interesting topic, and has been written well.

1. My expertises cover the Environmental Engineering, and treatment of water and wastewater. In this case, I would like to look at the paper from a different perspective. Identifying and characterizing pesticide use on 9,000 fields of organic agriculture would help us in preventing the spread of pesticides in the environments as well as in finding an efficient remediation technique.

In 2016, the global consumption of pesticides was 4.1 millions of tons, of which $1.4 \%$ was used in Oceania, 2.2\% in Africa, 11.8\% in Europe, 33.3\% in Americas and $51.3 \%$ in Asia . However, only 1\% of applied pesticides reached their target organisms with the remaining $99 \%$ entering soils and waters. Pesticide residues have been identified in various water bodies and soils worldwide, which threatens the humans and environments (https://doi.org/10.1016/j.chemosphere.2020.126646). Moreover, these organic contaminants would react with each other in the soil or water, and produce daughter compounds, which are more toxic than their parents (https://doi.org/10.1016/j.chemosphere.2016.06.047).

2. “For example, between 2000 and 2015, global organic agriculture grew from 15 million ha to 51 million ha2 and has since exceeded 73 million ha". I prefer to receive the updated detail in this sentence, since opinion of people about using organic crops has been improved during last years.

3. Table 3 provides the interesting results about comparison of soil quality in conventional farmlands and organic ones. There is no significant difference in soil quality, under cultivation of carrots and grapes, between organic and conventional lands. It means, we can encourage farmers and gardeners use the organic process in cultivation of carrots and grapes.

Thanks for giving me a chance to read this comprehensive study. 